\title{
DESIGN E ENVELHECIMENTO: UM ESTUDO SOBRE AÇÕES PROJETUAIS PARA A CONSTRUÇÃO DE UMA NOVA VELHICE
}

Dissertação apresentada ao Programa de Pósgraduação em Design da PUC-Rio como requisito parcial para obtenção do título de Mestre em Design.

Orientador: Prof ${ }^{a}$. Vera Maria Marsicano Damazio 
Renata de Andrade Marques Pereira

\section{DESIGN E ENVELHECIMENTO: UM ESTUDO SOBRE AÇÕES PROJETUAIS PARA A CONSTRUÇÃO DE UMA NOVA VELHICE}

Dissertação apresentada como requisito parcial para obtenção do grau de Mestre pelo Programa de Pós-graduação em Design do Departamento de Artes \& Design da PUC-Rio. Aprovada pela Comissão Examinadora abaixo assinada.

Profa. Vera Maria Marsicano Damazio

Orientadora

Departamento de Artes \& Design - PUC-Rio

Profa . Flávia Nizia da Fonseca Ribeiro Departamento de Artes \& Design - PUC-Rio

Prof ${ }^{a}$. Karine de Mello Freire Universidade do Vale do Rio Sinos

Profa. Denise Berruezo Portinari Coordenadora Setorial do Centro de Teologia e Ciências Humanas - PUC-Rio 
Todos os direitos reservados. É proibida a reprodução total ou parcial do trabalho sem autorização da universidade, da autora e do orientador.

\section{Renata de Andrade Marques Pereira}

Graduou-se em Desenho Industrial com habilitação em Projeto de Produto pela Universidade Federal do Rio de Janeiro (UFRJ) em 2011. É integrante do Laboratório Design Memória e Emoção da PUC-Rio desde 2012, pesquisando sobre Design \& Envelhecimento.

Ficha Catalográfica

Pereira, Renata de Andrade Marques

Design e envelhecimento: um estudo sobre ações projetuais para a construção de uma nova velhice / Renata de Andrade Marques Pereira ; orientadora: Vera Maria Marsicano Damazio. - 2014.

103 f. ; $30 \mathrm{~cm}$

Dissertação (mestrado)-Pontifícia Universidade Católica do Rio de Janeiro, Departamento de Artes e Design, 2014.

Inclui bibliografia

1. Artes e design - Teses. 2. Design e envelhecimento. 3. Nova velhice. 4. Representação social. I. Damazio, Vera Maria Marsicano. II. Pontifícia Universidade Católica do Rio de Janeiro. Departamento de Artes e Design. III. Título. 
Aos meus pais e às minhas avós, meus maiores exemplos de uma bela velhice. 


\section{Agradecimentos}

A minha querida orientadora Vera Damazio, por todo carinho, estímulo e parceria para a realização deste trabalho.

A minha mãe, por ser meu grande exemplo de nova velha.

Ao meu pai, pelo apoio incondicional a minhas escolhas.

A minhas avós, por serem meus exemplos e minha inspiração para este estudo.

A Thiago Born, por todo carinho, atenção e palavras de apoio.

Aos colegas do Labmemo, em especial Gabriel Leitão, Luiza Polli, Nathalia Bernardes, Marilia Ceccon e Silvia Nogueira, por todos os desafios enfrentados juntos, sempre embalados por muitas risadas.

A minha irmã Leticia e minha prima Gabriela, pela paciência e apoio incondicional.

A minhas amigas Ana Carolina Monteiro, Lisandre Ramos, Manuela Werneck, Mariana Império, Natália Treistman, Rennata Ramalho e Tainá Marques, por todo apoio e compreensão.

Aos professores que participaram da banca examinadora. 


\section{Resumo}

Pereira, Renata de Andrade Marques; Damazio, Vera. Design e Envelhecimento: um estudo sobre ações projetuais para a construção de uma nova velhice. Rio de Janeiro, 2014. 103p. Dissertação de Mestrado - Departamento de Artes e Design, Pontifícia Universidade Católica do Rio de Janeiro.

Este trabalho é uma investigação sobre o potencial do Design promover a diversidade, os ganhos e as possibilidades da velhice e partiu da constatação de que, apesar de plurais, os idosos são - em geral - tratados de forma homogênea e associados a produtos e serviços que ressaltam os aspectos negativos do avanço da idade, como bengalas e asilos. A abordagem sobre o tema se deu a partir de pesquisa bibliográfica e observação participante junto a idosos, e teve como principais condutores Simone de Beauvoir - autora de $A$ velhice, obra que retrata a condição do idoso sob o ponto de vista histórico, biológico e social -, Mirian Goldenberg - autora de A bela velhice - e Celso Sá - que trata da teoria da Representação Social: base para a elaboração de um exercício projetual que verifica como os idosos são representados socialmente por produtos e serviços. A investigação sobre o potencial do Design de contribuir com novas formas de ver e viver a velhice foi baseada em coleta de ações projetuais em prol de idosos realizada a partir da criação de grupos de interesse com pesquisadores do Centro de Estudo e Pesquisa do Envelhecimento e do International Longevity CenterBrazil e alunos de Ciências Sociais e de Design, entre outros. Os exemplos coletados foram organizados em categorias, com base no estudo de Mihaly Csikszentmihalyi sobre a forma que as pessoas constroem significados em seus ambientes familiares por meio de artefatos e nas perspectivas do Design Emocional identificadas por estudos desenvolvidos no laboratório Design Memória e Emoção. Observou-se a existência de dois grandes grupos: (1) o das "coisas de velho", que focam as limitações e perdas da velhice e tratam os idosos como um grupo homogêneo, e (2) o das "coisas do novo velho", que atendem a demandas variadas por meio de formas igualmente variadas. Ao final, conclui-se que, a construção da nova velhice está intrinsecamente ligada à visão daqueles que projetam produtos e serviços e às ações que pretende-se favorecer: dançar, namorar, trabalhar, viajar, viver. 


\section{Palavras-chave}

Design e Envelhecimento; Nova Velhice; Representação Social. 


\section{Abstract}

Pereira, Renata de Andrade Marques; Damazio, Vera. Design and Aging: a study of design actions for the construction of a new old age. Rio de Janeiro, 2014. 103p. MSc. Dissertation - Departamento de Artes e Design, Pontifícia Universidade Católica do Rio de Janeiro.

The present study is an investigation into the potential of Design to promote diversity, gains and possibilities of old age and began from the observation that although plural, the elderly are treated, in general, homogeneously and associated with products and services that highlight negative aspects of aging, such as canes and nursing homes. The approach on the theme occurred with literature research and participant observation with elderly people and had as main conductors Simone de Beauvoir - author of Old Age, book that depicts the condition of the elderly from historical, biological and social perspectives -, Mirian Goldenberg - author of The Beautiful Age - and Celso Sá who deals with the Social Representation Theory: basis for the elaboration of a projetual exercise that checks how the elderly are socially represented through products and services. The research about the potential of Design to contribute to new ways of seeing and living the old age was based on collecting examples of design actions on behalf of seniors, accomplished through the creation of interest groups with researchers from the Center for Research and Study of Aging and from the International Longevity Center-Brazil, students of Science social and Design, among others. The examples collected were organized into categories, based on the study by Mihaly Csikszentmihalyi on how people construct meaning in their familiar environments through artifacts, and prospects of Emotional Design identified by studies conducted in the lab Design Memory and Emotion. It was observed the existence of two major groups: (1) the "things of old people", focusing on the limitations and losses of old age and treat the elderly as a homogeneous group and (2) the "things of new old people" that meet varied demands through various forms also. Finally, it is concluded that the construction of the new old age is intrinsically linked to the vision of those who design products and services and actions intended to encourage: to dance, to date, to work, to travel, to live ... 


\section{Keywords}

Design and Aging; New Aging; Social Representation. 


\section{Sumário}

1 Introdução 19

1.1 Minha mãe, minha avó e eu: primeiros passos 20

1.2. Objetivos e questões norteadoras 21

$\begin{array}{ll}\text { 1.3. Metodologia } & 21\end{array}$

1.4. Principais autores $\quad 21$

1.5. Visão geral da dissertação 22

1.6. Sobre o título desta dissertação 23

2 Sobre velhice na sociedade atual 25

2.1. Estamos envelhecendo e vivendo cada vez mais 25

2.2. A Bela e a Nova Velhice 32

2.3. Os nomes da velhice: Velho?ldoso?Na melhor idade? 34

2.4. Velhice e diversidade: muitos e múltiplos 37

2.5. Velho é sempre o outro: Por que não nos vemos como 38 velhos?

2.6. Arco x Escada: envelhecer como um processo ascendente 42

2.7. Considerações parciais $\quad 44$

3 Sobre a mudança na representação social da velhice 46

3,1 Em busca das "coisas de velho 46

3.2. Contribuições da Psicologia Social 47

3.2.1. A teoria da Representação Social 48

3.2.2. A teoria do Núcleo Central 54

3.3. Um experimento sobre a mudança do Núcleo Central 59

3.4. Considerações parciais 66

4 O olhar do Design sobre o universo material dos idosos 68

4.1. Cartas na Mesa 68

4.2. Estudos classificatórios 70

4.3. Coisas de Velho x Coisas do Novo Velho 72

4.4. Categorias das Coisas do Novo Velho 76 
4.4.1. Autonomia 77

4.4.2. Sociabilidade $\quad 81$

4.4.3. Intergeracionalidade 83

4.4.4. Autoestima 85

4.4.5. Libertação 90

4.4.6. Segurança 93

4.5. Considerações parciais 96

5. Considerações finais 98

6. Referências bibliográficas 101 


\section{Lista de figuras}

Figura 1 - Porcentagem da população idosa em todo o mundo 26 nos anos de 2012 e 2050

Figura 2 - Reportagens especiais sobre o envelhecimento 28

Figura 3 - Participantes do curso sobre plataformas da Internet 33 durante as aulas de Skype

Figura 4 - Exemplo do Diário de velho 41

Figura 5 - Ilustração "o arco e a escada" 43

Figuras 6 e 7 - Processo de ancoragem das crianças diante de 49 um videogame antigo.

Figura 8 - Exemplo de pictogramas 50

Figura 9 - Exemplo do uso da bengala no pictograma 56 do idoso no Brasil.

Figura 10 - Exemplo de produtos para idosos. 56

Figura 11 - Imagem ilustrativa da representação 58 social da velhice

Figura 12 - Imagem ilustrativa da representação social 59 da nova velhice

Figura 13 - Folhas utilizadas no primeiro experimento. 60

Figura 14 - Imagens com maior frequência do ensaio

Figura 15 - Imagens com maior frequência do ensaio 62

Figura 16- Folhas utilizadas no segundo experimento 64

Figura 17 - Imagens com maior frequência do ensaio 65

Figura 18 - Imagens com maior frequência do ensaio 66

Figura 19 - Algumas das cartas desenvolvidas e 69 utilizadas nos ensaios de organização.

Figura 20 - Exemplos de pictogramas que representam

o idoso na Espanha, Turquia, Itália e Brasil, nessa ordem.

Figuras 21 e 22 - Bengalas com forma "clássica" 73

Figuras 23, 24 e 25 - Linha de bengalas criadas pelos

designers Francesca Lanzavecchia e Hunn Wai

Figuras 26 e 27 - Comparação entre o babador de forma 
Figuras 28 e 29 - Comparação entre a fralda geriátrica e

a calcinha geriátrica

Figuras 30 e 31 - Comparação entre o porta-remédios de

Forma "clássica" e o porta-remédios escultural

Figuras 32 e 33 - Comparação entre o andador geriátrico

76

e o andador Trikka

Figuras 34 e 35 - Comparação entre os pictogramas do idoso 76

Figura 36 - Sistema Liftware com o encaixe de colher $\quad 78$

Figura 37 - Características do Liftware $\quad 78$

Figura 38 - Exemplo do manuseio dos talheres especiais $\quad 79$

desenvolvidos pela empresa "Dining with Dignity".

Figuras 39, 40, 41 e 42 - Blusa com abertura traseira;

79

Vestido com abertura traseira.

Figuras 43 e 44 - Aparelhos de assistência da Telehelp para $\quad 80$ fora e dentro de casa

Figura 45 - Simulação do serviço de entrega Overdelivery. $\quad 82$

Figura 46 - Imagem do site Novas emoções com depoimento 83

de usuários

Figura 47 e 48 - Membros de diferentes idades da orquestra

de New Jersey

Figuras 49 e 50 - Workshop de arte urbana realizado

pelo projeto "Lata65"

Figura 51,52 e 53 - Imagens dos webchats

85

Figura 54,55 e 56 - O fotógrafo Ari Cohen e seus fotografados 86

do blog Advanced Style

Figuras 57 e 58 -Integrantes da família de palhaços Labelle. $\quad 87$

Figuras 59 e 60 - Palhaços interagindo com idosos $\quad 87$

Figuras 61 e 62 - Imagens de competições durante os $\quad 88$

Jogos Regionais do Idoso

Figuras 63 e 64 - Trabalhos enviados para a edição de $2013 \quad 88$

do concurso Talentos da Maturidade.

Figuras 65, 66, 67 e 68 - Imagens do projeto Mamika

Figuras 69, 70, 71 e 72 - Exemplos do look do dia de Ali 
Figuras 73 e 74 - Liu Xianping vestido com roupas femininas 92 da grife da neta

Figuras 75 e 76 - Imagens da banda The Zimmers 92

Figuras 77 e 78 - Imagens de Mamy Rock, a $D J$ idosa 93

Figuras 79, 80, 81 e 82 - Tênis Aetrex 94

Figuras 83, 84, 85 e 86 - Detalhe da campanha e imagens dos 95 cartazes da campanha Up in years

Figura 87 - Interface da tecnologia Doro 95

Figura 88 - llustração indicando a mistura entre as diferentes 96 categorias 


\section{Lista de gráficos}

Gráfico 1 - Indicativo da proporção de cada faixa etária nos

anos de 2000, 2013 e 2050

Gráfico 2 - Frequência de respostas dos participantes com

61

menos de 30 anos

Gráfico 3 - Frequência de respostas dos

62

participantes com menos de 30 anos

Gráfico 4 - Frequência de respostas dos participantes

65

entre 30 e 75 anos

Gráfico 5 - Frequência de respostas dos participantes

66

entre 30 e 75 anos 


\section{Lista de tabelas}

Tabela 1 - Adjetivos dos velhos sub e super 


\section{Lista de quadros}

Quadro 1 - Três condições de existência da nova

representação social da velhice 
Não importa se a estação do ano muda. Se o século vira, se o milênio é outro. Se a idade aumenta. Conserva a vontade de viver. Não se chega a parte alguma sem ela.

Fernando Pessoa 